02,12

\title{
Сверхпроводимость, инициированная электрическим полем в высокотемпературном сверхпроводнике при $T>T_{c}$
}

\author{
(C) М.А. Кожушнер ${ }^{1}$, В.С. Посвянский ${ }^{1}$, Б.В. Лидский ${ }^{1}$, В.Л. Боднева ${ }^{1}$, Л.И. Трахтенберг ${ }^{1,2}$ \\ ${ }^{1}$ Федеральный исследовательский центр химической фризики им. Н.Н. Семенова, РАН, \\ Москва, Россия \\ ${ }^{2}$ Московский государственный университет им. М.В. Ломоносова, \\ Москва, Россия \\ E-mail: kozhushner@gmail.com
}

Поступила в Редакцию 17 марта 2020 г.

В окончательной редакции 17 марта 2020 г.

Принята к публикации 16 апреля 2020 г.

Развита теория возникновения сверхпроводимости в пластине высокотемпературного сверхпроводника под действием перпендикулярного ей электрического поля, когда при температуре $T<T_{c}^{\max }$ концентрация носителей лежит вне области существования сверхпроводимости. Расчет проведен для слоя лантанстронциевого купрата в полях $10^{-1} \mathrm{~V} / \mathrm{nm} \geq E \geq 10^{-2} \mathrm{~V} / \mathrm{nm}$ при разных температурах и концентрациях дырок. Показано, что вблизи поверхности пластины возникает квазидвумерный сверхпроводящий слой толщиной несколько ангстрем, причем толщина этого слоя не зависит от величины поля, а зависит лишь от температуры и концентрации дырок.

Ключевые слова: квазидвумерность, сверхпроводимость, дырки, концентрация, электрическое поле.

DOI: $10.21883 /$ FTT.2020.08.49595.059

\section{1. Введение}

После открытия высокотемпературной сверхпроводимости (ВТСП) [1] стало ясно, что проводимость и сверхпроводящий переход обеспечивается куперовским спариванием между дырками или электронами, образующимися в купратных оксидах из-за частичной замены элементов в их составе (см. обзор [2]). Валентность заменяющего элемента должна быть больше или меньше, чем у заменяемого. Вследствие этого возникают свободные дырки либо электроны. Оказалось, что для всех купратов область существования сверхпроводимости на плоскости с ординатой — „температура“ и абсциссой — „концентрация носителей“ находится под колоколообразной кривой — „квазипараболой“ ${ }^{\prime}$ с основанием на оси абсцисс, а ордината вершины - максимальное значение температуры сверхпроводящего перехода $T_{c}=T_{c}^{\max }$ [3]. Что касается механизма спаривания носителей тока, то среди нескольких предложенных механизмов [2] спинполяронная связь кислородных дырок и спинов ионов меди, разработанная в работах [4,5], дает возможность найти энергетическую структуру дырок в плоской зоне Бриллюэна в купрате $\mathrm{La}_{2-x} \mathrm{Sr}_{x} \mathrm{CuO}_{4}$.

Связь температуры сверхпроводящего перехода с плотностью носителей заряда и с механизмом их спаривания дает возможность повлиять на $T_{c}$ внешним электрическим полем. Еще до открытия ВТСП такая попытка была предпринята в экспериментальной работе [6], где в тонких пленках In и Sn при гелиевых температурах было получено изменение $T_{c}$ на величину $\sim 10^{-4} \mathrm{~K}$ в электрическом поле $2.6 \cdot 10^{5} \mathrm{~V} / \mathrm{sm}$. В статьях [7,8] было предложено влиять на $T_{c}$ в тонких полосках ВТСП $\mathrm{YBa}_{2} \mathrm{Cu}_{3} \mathrm{O}_{x}$ и $\mathrm{Bi}_{2} \mathrm{Sr}_{2} \mathrm{CaCu}_{2} \mathrm{O}_{8}$ за счет изменения плотности носителей при проникновении в нее электронов из металлических обкладок под действием электрического поля. Влияние электрического поля на механизм спаривания носителей в ВТСП обсуждалось с использованием уравнения Bethe-Salpeter [9]. Одновременное влияние сильного поля $\left(\sim 10^{8} \mathrm{~V} / \mathrm{sm}\right)$ на механизм спаривания и изменение концентрации носителей за счет прихода из металла в тонкую пластину ВТСП $\left(\mathrm{YBa}_{2} \mathrm{Cu}_{3} \mathrm{O}_{7-y}\right.$ и $\left.\mathrm{Bi}_{2} \mathrm{Sr}_{2} \mathrm{CaCu}_{2} \mathrm{O}_{8+x}\right)$ рассматривалось в статье [10]. Обзор работ, выполненных до 2002 г., посвященных влиянию электрического поля на сверхпроводимость, представлен в публикации [11].

В данной работе, в отличие от статей $[7,8]$, рассматривается возникновение СП-состояния в приповерхностном слое изолированной пластины ВТСП (см. рис. 1) при температуре $T>T_{c}$ под действием перпендику-

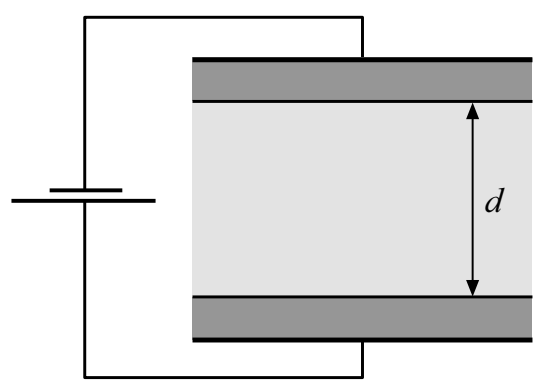

Pис. 1. Схема подключения ВТСП-пластины ( $\square)$ толщиной $d$ через изолирующие прокладки $(\square)$. 
лярного к пластине электрического поля, при котором происходит изменение плотности носителей тока. Такой расчет может быть сделан для лантан-стронциевого купрата, т.к. для него недавно была рассчитана энергетическая структура зоны носителей - дырок [4,5] и вычислена зависимость $T_{c}$ от плотности носителей, совпадающая с экспериментальной [3].

При воздействии электрического поля у одной стороны пластины оксида должна увеличиваться плотность дырок. Если без поля их средняя плотность меньше критической плотности при данной температуре, то из-за поля плотность дырок вблизи края пластины может стать больше критической, возникнет сверхпроводимость, и вдоль полоски потечет сверхпроводящий ток, т. е. можно говорить о сверхпроводящем транзисторе.

\section{2. Формулировка задачи}

Рассматривается слой купрата $\mathrm{La}_{2-x} \mathrm{Sr}_{x} \mathrm{CuO}_{4}$ (ВТСП с максимальной температурой сверхпроводящего перехода $T_{c}^{\max } \cong 40 \mathrm{~K}$ при концентрации дырок $x_{\max } \cong 0.14$ ) со средней концентрацией дырок $x_{0}$, толщиной $d=200$ (здесь и далее все физические величины задаются в а.е.), с диэлектрической проницаемостью $\eta$ при температуре $T$. На плоскости $(T, x)$ прямая $T=\mathrm{const}$ при $T<T_{c}^{\max }$ пересекает критическую „квазипараболу, в двух точках с абсциссами $x_{1}(T)$ и $x_{2}(T)$, так что сверхпроводимость при температуре $T$ осуществляется при средней концентрации дырок $x_{0}$, когда $x_{1}(T)<x_{0}<x_{2}(T)$. Рассмотрим два случая, когда в отсутствии электрического поля нет сверхпроводимости: 1) $x_{0}<x_{1}(T)$ и 2) $x_{0}>x_{2}(T)$. Перпендикулярно слою прикладывается электрическое поле, т. е. между границами слоя задается разность потенциалов $U$ (рис. 1$)$. Тогда плотность дырок становится больше в области малых потенциалов и меньше в области больших, т.е. в этих областях либо в первом, либо во втором случае может образоваться сверхпроводящий слой. Выяснению зависимости толщины этого слоя от величины электрического поля посвящена данная работа.

\section{3. Свободная энергия сверхпроводящего слоя}

Распределение дырок в слое находится при минимизации свободной энергии системы $F$ по плотности дырок $n(z)$, где ось $z$ перпендикулярна поверхности слоя. Свободная энергия системы записывается в виде

$$
F=F_{1}+F_{2},
$$

где $F_{1}-$ свободная энергия неоднородного дырочного газа, а $F_{2}$ - электростатическая энергия. Первое слагаемое в правой части выражения (1) имеет вид

$$
F_{1}=\int_{0}^{d} d z F_{1}(z),
$$

$$
\begin{aligned}
& F_{1}(z)=-\frac{2 k T}{\pi^{2} \Omega} \int_{0}^{\pi} \int_{0}^{\pi} d p_{x} d p_{y} \\
& \times \ln \left(1+\exp \left\{\frac{\mu(z)-\varepsilon\left(p_{x}, p_{y} ; n(z)\right)}{k T}\right\}\right)+\mu(z) n(z) .
\end{aligned}
$$

Формула (3) характеризует изменение плотности свободной энергии $F_{1}(z)$ по координате $z, \Omega-$ объем элементарной ячейки, равный 1180 a.u. Поскольку дырки в кристалле купрата движутся в сверхпроводящих плоскостях квадратов $\mathrm{CuO}_{4}$, симметричных относительно осей абсцисс и ординат, перпендикулярных сторонам квадрата, то энергетическая зона в обратной двумерной квадратной решетке одинакова в каждом из 4-х секторов обратной решетки [5]. Поэтому интегрирование по импульсам $p_{x}, p_{y}$ в формуле (3) проводится только в квадрате обратной решетки $\left[0 \leq p_{x} \leq \pi / a ; 0 \leq p_{y} \leq \pi / a\right]$, где $a-$ сторона квадрата $\mathrm{O}_{4}$ в элементарной ячейке купрата, а результат интегрирования умножается на 4.

Локальный химический потенциал $\mu(z)$ определяется плотностью $n(z)$ с помощью равенства

$$
\begin{aligned}
n(z)= & \frac{2}{\pi^{2} \Omega} \int_{0}^{\pi} \int_{0}^{\pi} d p_{x} d p_{y} \\
& \times \frac{1}{1+\exp \left\{\left(\varepsilon\left(p_{x}, p_{y} ; n(z)\right)-\mu(z)\right) / k T\right\}} .
\end{aligned}
$$

Плотность свободных дырок $n(z)$ задается в виде

$$
n(z)=x(z) \cdot \Omega^{-1}=x(z) \cdot 0.85 \cdot 10^{-3},
$$

где $x(z)$ - концентрация дырок $0<x(z)<1$.

Выражение для показателя экспоненты в формулах (3), (4) для сверхпроводника известно [12]. С учетом зависимости величины сверхпроводящей щели $\Delta$ от координат в обратной ячейке [5], это выражение имеет вид

$$
\begin{aligned}
\mu(z) & -\varepsilon\left(p_{x}, p_{y} ; n(z)\right)=\left\{\left[\mu(z)-E_{1}\left(p_{x}, p_{y} ; n(z)\right)\right]^{2}\right. \\
& \left.+\left[\Delta_{0}(T, n(z))\left(\cos p_{x}-\cos p_{y}\right)\right]^{2}\right\}^{1 / 2}
\end{aligned}
$$

Зависимости энергии дырок $E_{1}\left(p_{x}, p_{y} ; n(z)\right)$ от координат в обратной решетке и плотности дырок, а также величина сверхпроводящей щели в спектре дырок $\Delta_{0}(T, n(z))$ от температуры и плотности дырок найдены в работах $[4,5]$ и в дальнейших расчетах будут задаваться численно. Щель $\Delta_{0}(T, n(z))$ отлична от 0 в ограниченной области температур и концентраций, а вне этой области в формулах (3), (4), исходя из выражения (6), $\varepsilon\left(p_{x}, p_{y} ; n(z)\right)=E_{1}\left(p_{x}, p_{y} ; n(z)\right)$. 
Электростатическая энергия определяется уравнением [13]:

$$
F_{2}=\frac{\eta}{8 \pi} \int_{0}^{d}\left(\frac{d \varphi}{d z}\right)^{2} d z,
$$

где потенциал $\varphi$ подчиняется уравнению Пуассона

$$
\frac{d^{2} \varphi}{d z^{2}}=-\frac{4 \pi}{\eta}\left[n(z)-n_{-}\right]
$$

с граничными условиями

$$
\varphi(0)=0, \quad \varphi(d)=U .
$$

Здесь $U$ и $n_{-}$- заданные разность потенциалов на краях сверхпроводящей пластины и средняя плотность анионов. Естественно, выполняется закон сохранения заряда

$$
\int_{0}^{d} d z\left[n(z)-n_{-}\right]=0 .
$$

Плотность анионов записывается в виде

$$
n_{-}=x_{0} \cdot 0.85 \cdot 10^{-3},
$$

где $x_{0}-$ заданная средняя концентрация анионов, а $0.85 \cdot 10^{-3}$ - обратный объем элементарной ячейки.

В выражении (3) энергия дырок $\varepsilon\left(p_{x}, p_{y} ; n(z)\right)$ описывается формулой (6), т. к. сверхпроводящие пары не дают вклада в свободную энергию. В то же время, величина $n(z)$ в уравнении Пуассона (8) и в законе сохранения заряда (9) включает в себя как свободные дырки, так и сверхпроводящие пары, поскольку сверхпроводящие пары дырок имеют заряд.

\section{4. Метод решения}

Математически задача поиска распределения дырок $n(z)$, дающего минимум свободной энергии, является задачей вариационного исчисления с ограничением [14]. Для ее решения необходимо вычислить вариацию функционала $\delta F$. Приравняв вариацию нулю, получим соотношение между химическим и электрическим потенциалами $\mu+\varphi=C$ (Здесь $C$ произвольная постоянная). В результате плотность дырок становится функцией электрического потенциала. Окончательно задача сводится к решению уравнения второго порядка (8) относительно неизвестной функции $\varphi$ с условиями (8a). Постоянная $C$ определяется из условия закона сохранения заряда (10). Краевая задача (8), (8a) решалась численно методом установления с использованием неявной разностной схемы [15].

\section{5. Толщина сверхпроводящего слоя}

Экспериментальная кривая $T_{c}(x)$ для $\mathrm{La}_{2-x} \mathrm{Sr}_{x} \mathrm{CuO}_{4}$ [3], ограничивающая область существования сверхпроводимости на плоскости $(T, x)$, может быть с неплохой точностью представлена в виде

$$
T_{c}(x)=40-4000(x-0.15)^{2} .
$$

Нетрудно видеть, что максимальное значение температуры сверхпроводящего перехода $T_{c}^{\max }=40 \mathrm{~K}$, а область концентраций дырок, при которой существует сверхпроводимость при температурах $T<T_{c}^{\max }$, определяется неравенством

$$
0.15-\sqrt{\frac{T_{c}^{\max }-T}{4000}}<x<0.15+\sqrt{\frac{T_{c}^{\max }-T}{4000}} .
$$

Проведен расчет зависимости концентрации $x$ от координаты $z$ (по толщине слоя) при действии поля, направленного вдоль $z$, при различных температурах $T<T_{c}^{\max }$ и средних концентрациях $x_{0}$, лежащих вне помежутка (11a). Рассматривались различные разности потенциалов между сторонами слоя: $U=2 \cdot 10^{-3}-2 \cdot 10^{-2}(0.054 \mathrm{~V}-0.54 \mathrm{~V})$ при различных температурах и средних концентрациях дырок: 1) $T=30 \mathrm{~K}$ (область сверхпроводимости $[0.2>x>0.1]$ ), $\left.x_{0}=0.09, x_{0}=0.08, x_{0}=0.21 ; 2\right) T=20 \mathrm{~K}$ (область сверхпроводимости $[0.22>x>0.08]), x_{0}=0.075$.

При действии поля плотность дырок должна увеличиваться в области малого потенциала и уменьшаться в области большого потенциала. Поэтому при $x_{0}<0.15-\sqrt{\left(T_{c}^{\max }\right) / 4000}$ сверхпроводимость при достаточной величине разности потенциалов появится около отрицательного полюса батареи, а при $x_{0}>0.15+\sqrt{\left(T_{c}^{\max }\right) / 4000}-$ у положительного полюса.

На рис. 2 показаны зависимости концентрации дырок $\mathrm{x}$ от координаты при $x_{0}=0.09, T=30 \mathrm{~K}$ для двух разностей потенциалов на сторонах пластины $U=0.01$; $0.02(0.272 \mathrm{~V} ; 0.544 \mathrm{~V})$. Как и должно быть в проводнике, плотность дырок изменяется только у краев пластины, оставаясь неизменной в толще пластины. Интервал концентраций, в котором существует сверхпроводимость при $T=30 \mathrm{~K}$, согласно условиям (11a) равен $[0.2>x>0.1]$. Толщина сверхпроводящего слоя $\delta_{S C} \sim 6.7(3.5 \AA)$ и, как показывают расчеты, не зависит от величины приложенного поля, а зависит только от $x_{0}$ и температуры. Так при $x_{0}=0.08, T=30, \delta_{S C} \sim 4(2 \AA)$, а при $x_{0}=0.075, T=20 \mathrm{~K}, \delta_{S C} \sim 7.3(3.9 \AA)$.

Если средняя концентрация дырок $x_{0}$ больше верхней границы интервала существования сверхпроводимости, то сверхпроводящий слой возникает у противоположной стороны пластины в области бо́льшего электростатического потенциала - при $x_{0}=0.21, \quad T=30$, $\delta_{S C} \sim 6.2(3.3 \AA)$ и $\delta_{S C}$ не зависит от напряжения. От приложенного напряжения зависит лишь расстояние $\delta_{0}$ от края пластины до начала области сверхпроводимости. При $x_{0}=0.09, T=30, \delta_{0}=0$ при $U \sim 0.0015(0.04 \mathrm{~V})$, 


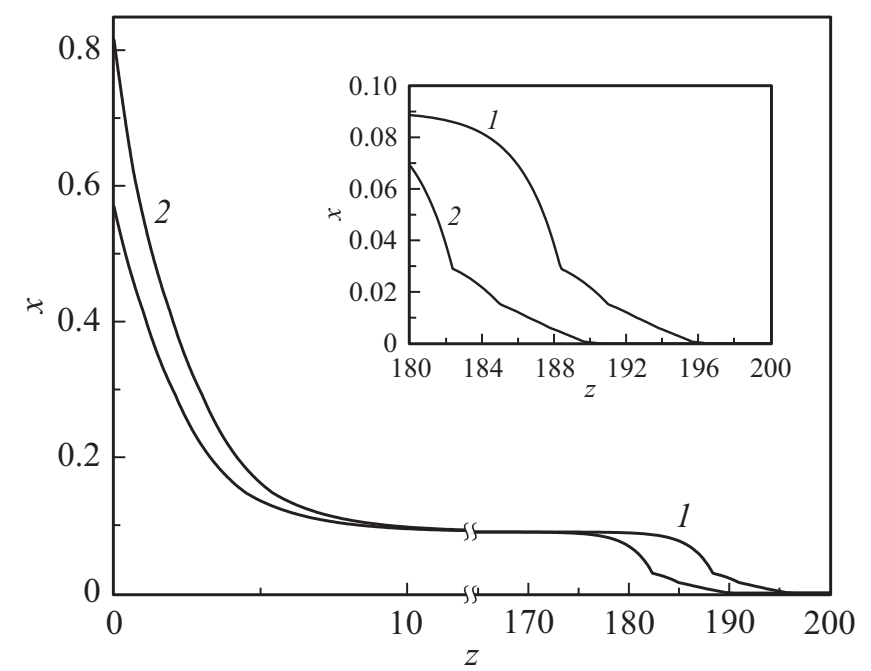

Рис. 2. Зависимости плотности дырок $x$ от координаты $z$ при $x_{0}=0.09, T=30 \mathrm{~K}$ для двух разностей потенциалов на сторонах пластины $U=0.01(0.272 \mathrm{~V})$ (кривая 1$)$ и $U=0.02(0.544 \mathrm{~V})$ (кривая 2).

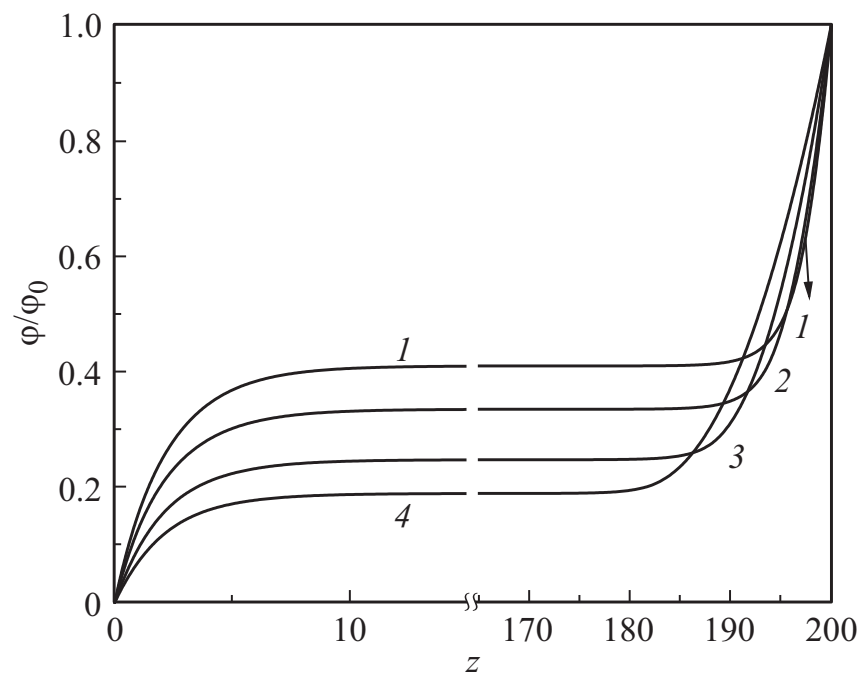

Рис. 3. Относительное изменение потенциала внутри пластины при $x_{0}=0.09, T=30 \mathrm{~K}$ для различных значений напряжений на краях пластины $U=0.002(0.0544 \mathrm{~V})$ (кривая 1$)$, $U=0.004(0.1088 \mathrm{~V})$ (кривая 2), $U=0.01(0.272 \mathrm{~V})$ (кривая 3$), U=0.02(0.544 \mathrm{~V})$ (кривая 4).

и $\delta_{0}=4(2 \AA)$ при $U=0.02(0.54 \mathrm{~V})$. Таким образом, при больших напряжениях сверхпроводящий слой находится внутри пластины недалеко от ее края.

Электростатический потенциал изменяется внутри пластины, естественно, только вблизи краев, но по-разному - в зависимости от величины приложенного напряжения. На рис. 3 показано относительное изменение потенциала внутри пластины при $x_{0}=0.09$, $T=30 \mathrm{~K}$ для различных значений напряжения на краях пластины. Видно, что чем больше приложенное напряжение, тем больше относительная разница в падении потенциала вблизи разных краев пластины. Это связано с относительно небольшой средней концентрацией зарядов в сверхпроводнике.

Суммарный размер областей изменения плотности носителей (дырок), совпадающих с областями изменения потенциала (или, иначе, с областями, где электрическое поле не равно нулю), существенно меньше полной толщины пластины. Это означает, что размер и положение областей, где возникает сверхпроводимость при данном напряжении на сторонах пластины, не зависит от толщины пластины $d$, если $d \geq 100(\sim 5 \mathrm{~nm})$. Таким образом, в любой такой пластине ВТСП при $T<T_{c}$ и концентрации дырок $x$, лежащей вне сверхпроводящего промежутка (11a), при поперечном напряжении $U$, большем нескольких сотых вольта, возникает приповерхностный сверхпроводящий слой. Как следует из рис. 2, чем больше концентрация $x$ отстоит от границ сверхпроводящего промежутка (11a), тем тоньше слой возникающей сверхпроводимости. Поскольку толщина возникающего сверхпроводящего слоя составляет несколько А̊, т.е. атомной толщины, то это значит, что под действием поля возникает квазидвумерная сверхпроводимость.

Возможность квазидвумерной сверхпроводимости обсуждалась еще в работе [16]. Двумерная сверхпроводимость наблюдалась в интерфейсе между двумя соприкасающимися оксидами [17]. Теоретическое и экспериментальное изучение двумерной сверхпроводимости представлено в обширном обзоре [18]. В недавних работах $[19,20]$ была также обнаружена квазидвумерная сверхпроводимость: в двойном слое атомов индия и молекул Fе-фталоцианин на поверхности кремния [19] и в промежутке между слоями изолятора $\mathrm{La}_{2} \mathrm{CuO}_{4}$ и ферроэлектрика $\mathrm{Ba}_{0.8} \mathrm{Sr}_{0.2} \mathrm{TiO}_{3}[20]$.

Очевидно, что возникновение квазидвумерной сверхпроводимости под действием электрического поля происходит в слое любого ВТСП при температуре с концентрацией носителей вне области существования сверхпроводимости. Возникновение сверхпроводимости, т. е. продольных токов в пластине ВТСП, под действием малых поперечных напряжений является физической основой сверхпроводящего транзистора.

\section{6. Заключение}

В работе теоретически показана возможность создания квазидвумерной сверхпроводимости - сверхпроводящего слоя атомной толщины вблизи поверхности пластины недодопированного или передопированного сверхпроводящего купрата, если температура меньше $T_{c}^{\max }$. Для этого к поверхностям пластины, толщина которой больше нескольких нанометров, прикладывается напряжение порядка десятых вольта, т. е. такая структура может выполнять роль ВТСП-транзистора. Расчет проведен для $\mathrm{La}_{2-x} \mathrm{Sr}_{x} \mathrm{CuO}_{4}$, для которого известна энергетическая структура дырочной зоны и форма кривой $\left(T_{c}, x\right)$. Вместе с тем, такой эффект возникновения квазидвумерной сверхпроводимости под действием электрического 
поля возможен в любом ВТСП-материале, так как при этом всегда существует ограниченная область $\left(T_{c}, x\right)$, в которой может появиться сверхпроводящий слой.

\section{Благодарности}

Авторы выражают глубокую благодарность В.В. Валькову, Д.М. Дзебисашвили, Л.Г. Мамсуровой, К.С. Пигальскому и Н.Г. Трусевичу за плодотворные обсуждения работы.

\section{Финансирование работы}

Работа выполнена в рамках Государствен-
в (AAAA-A18-118012390045-2), а также при поддержке Российского фонда фундаментальных исследований (гранты №№ 18-03-00528, 17-29-10016).

\section{Конфликт интересов}

Авторы заявляют, что у них нет конфликта интересов.

\section{Список литературы}

[1] J.G. Bednorz, K.A. Müller. Z. Phys. B 4, 189 (1986).

[2] B. Keimer, S.A. Kivelson, M.R. Norman, S. Uchida, J. Zaanen. Nature 518, 179 (2015).

[3] A. Ekert, R. Jozsa. Rev. Mod. Phys. 68, 733 (1996).

[4] V.V. Val'kov, D.M. Dzebisashvili, A.F. Barabanov. Phys. Lett. A 379, 421 (2015).

[5] В.В. Вальков, Д.М. Дзебисашвили, А.Ф. Барабанов. Письма ЖЭТФ 104, 745 (2016).

[6] R.E. Glover, M.D. Sherill. Phys. Rev. Lett. 5, 248 (1960).

[7] S.I. Shkuratov. J. Vacuum Sci. Technology 11, 353 (1993).

[8] S. Sakai. Phys. Rev. B 47, 9042 (1993).

[9] K. Moravets. Phys. Rev. B 66, 172508 (2002).

[10] P. Konsin, B. Sorkin. Phys. Rev. B 58, 5795 (1998).

[11] C. H. Ahn, J.-M.Triscone, J. Mannhart. Nature 424, 1015 (2003).

[12] Л.Д. Ландау, Е.М. Лифшиц.Статистическая физика. Наука, М. (1995). $624 \mathrm{c}$.

[13] Л.Д. Ландау, Е.М. Лифшиц. Электродинамика сплошных сред. Наука, М. (1982). 606 с.

[14] I.M. Gelfand, S.V. Fomin. Calculus of variations. Prentice-Hall, Inc., Englewood Cliffs, N. J. (1963). 242 c.

[15] С.К. Годунов, В.С. Рябенький. Разностные схемы. Наука, M. (1977). $442 \mathrm{c}$.

[16] T.M. Rice. Phys. Rev. 140, 1889 (1965).

[17] N. Reyren, S. Thiel, A.D. Caviglia, L.F. Kourkoutis, G. Hammerl, C. Richter, C.W. Schneider, T. Kopp, A.-S. Rüetschi, D. Jaccard, M. Gabay, D.A. Muller, J.-M.Triscone. J. Mannhart. Science 317, 1196 (2007).

[18] T. Uchihashi. Superconducter Sci. Tekhnology 30, 1 (2016).

[19] T. Uchihashi, S. Yoshizava, E. Minamitami, S. Watanabe, Y. Takagi, T. Yokoyama. Mol. Syst. Des. Eng. 4, 511 (2019).

[20] D.P. Pavlov, R.R. Zagidullin, V.M. Mikhailov, V.V. Kabanov, T. Adachi, T. Kawamata, Y. Koike, R.F. Mamin. Phys. Rev. Lett. 122, 237001 (2019).

Редактор К.В. Емщев 